\title{
Management of Neonatal Ovarian Cysts: Clinical Aspects
}

Francesco Saverio Camoglio, Federica Bianchi, Marta Peretti, Simone Patanè, Valentina Spigo and Nicola Zampieri ${ }^{*}$ Woman and Child Hospital, Pediatric Surgical Unit, University of Verona, Via S. Francesco, 22, 37129 Verona VR, Italy
Abstract
Introduction: Abdominal cysts are uncommon in neonate during prenatal ultrasound. Only 5\% is represented by ovarian cysts. Treatment options depend on the risk of complications. The aim of this study is to report the authors' experience and describe the most appropriate management in case of suspected ovarian cysts.
Materials and Methods: A retrospective analysis was carried out on all suspected ovarian cysts detected with prenatal ultrasound from January 2003 and December 2016. After birth all newborns underwent blood tests and ultrasound (US); cysts were classified into simple and complex. The authors described a conservative management or surgical treatment, depending on the signs and symptoms of the newborn, US cyst size and US cyst classification (simple vs complex).
Results: 98 patients with diagnosis of ovarian cyst were included in the study. US scan identified 73 simple cysts and 24 complex cysts. 31 patients underwent surgery. Twenty patients were treated with sparing surgery.
Discussion: In general, postnatal choice of treatment is depending on the size and appearance of the cyst. Complex cysts usually do not require urgent surgery because they generally represent the outcome of an antenatal ovarian torsion. Minimally invasive surgery is the gold standard for these patients.

\section{Publication History:}

Received: July 10, 2017

Accepted: September 18, 2017

Published: September 20, 2017

Keywords:

Ovarian, Cysts, Laparoscopy

\section{Introduction}

Before the routine use of ultrasound (US) scan, only large abdominal cysts palpable on physical examination could be detected. Thanks to routine ultrasound scan during pregnancy, detection has increased considerably and has been estimated at 1/500-1000 live births [1].

Of these only $5 \%$ is represented by ovarian cysts which incidence is about $1 / 2500$ pregnancies [2].

US scan is the gold standard for diagnosis, though it is suggested by others to perform Magnetic Resonance Imaging (MRI) or videolaparoscopy to differentiate ovarian cysts from other abdominal masses.

Cysts were classified on Nussbaum's ultrasound criteria into simple and complex. Other abdominal cysts could be a "free-floating" due to autoamputation of ovarian cysts [3-4].

As a result of exposure to maternal hormones, most neonatal ovarian cysts spontaneously resolve during the first months of life.

More recent studies have shown that simple cysts $<5 \mathrm{~cm}$ on postnatal imaging will likely spontaneously resolve [5].

Surgical treatment is indicated only in complex cysts or in simple cysts $>5 \mathrm{~cm}$ in order to avoid further complications and to preserve residual ovary, especially in presence of bilateral cysts [5 ].

The aim of this study is to report the authors' experience and describe the optimal management in case of suspected ovarian cyst.

\section{Materials and Methods}

In this study, retrospective analysis was carried out on all suspected ovarian cysts detected with prenatal ultrasound from January 2003 and December 2016.
Prenatal diagnosis was made using real-time, high resolution scanning with a $3.5 \mathrm{MHz}$ convex probe and adaptor.

After birth, within the first week of life, all patients underwent abdominal US scans in order to confirm or identify the nature of the cyst.

Inclusion criteria for the study were: full term female baby without other patologies; normal weight and size for gestational age; normal urinary and gastrointestinal tracts; regular pregnancy; absence of foetal distress during labor.

Cysts were classified on Nussbaum's ultrasound criteria into simple and complex [3].

Simple cyst was described as round, anechoic, unilocular, with fine wall and no or only one septation. Complex cyst was described as thick walled with heterogeneous elements of echogenicity such as multiple septations, mobile internal echoes (clots or debris), solid appearance or fluid-fluid levels.

The choice between conservative management and surgical treatment was based on the signs/symptoms of the newborn, US cyst size and US cyst classification (simple vs complex).

Conservative management included US every 2-6 weeks until cyst spontaneous regression.

"Corresponding Author: Dr. Nicola Zampieri, Woman and Child Hospital, Pediatric Surgical Unit, University of Verona, Via S. Francesco, 22, 37129 Verona VR, Italy, Tel: +39045 8124916, Fax: +39 045 8124662; E-mail: dr.zampieri@libero.it

Citation: Camoglio FS, Bianchi F, Peretti M, Patanè S, Spigo V, et al. (2017) Management of Neonatal Ovarian Cysts: Clinical Aspects. Int J Gynecol Clin Pract 4: 130. doi: https://doi.org/10.15344/2394-4986/2017/130

Copyright: (c) 2017 Camoglio et al. This is an open-access article distributed under the terms of the Creative Commons Attribution License, which permits unrestricted use, distribution, and reproduction in any medium, provided the original author and source are credited. 
For all those patients who underwent surgery, attempts were made to preserve normal ovarian tissue on the ipsilateral side whenever possible. All patients underwent blood exams (serum levels of alphafetoprotein, Beta-hCG and CA-125) in order to avoid tumoral masses.

All patients were treated with minimally invasive surgery, with two possible approaches, based on surgeons' preference: standard laparoscopy (3 trocars, 3 or $5 \mathrm{~mm}$ ), or laparoscopic-assisted transumbilical procedure (1 umbilical trocar, $10 \mathrm{~mm}$ ). Puncture and percutaneous evacuation of cyst under vision was one of the option during surgery.

Statistical analysis was performed using the Chi-Square and Fisher exact tests with a $P$ value less than 0.05 considered as significant.

\section{Results}

During the study period 105 patients were considered; 8 patients were excluded due to a post-natal diagnosis of mesenteric cyst. After reviewing medical charts, one patient was included because transferred from another hospital for abdominal unknown cyst. At the end 98 patients with diagnosis of ovarian cyst were included in the study.

Only one patient was symptomatic, with signs and symptoms of bowel obstruction; US scan showed a complex ovarian cyst of $7 \mathrm{~cm}$ and for this reason the patient underwent surgery within the first day of life.

Within the first week of life all the newborns underwent US scan and were identified 73 simple cysts and 24 complex cysts, following the Nussbaum's classification.

Spontaneous regression of simple cysts was observed in 67 cases (92\% of simple cysts) within 6 months afer birth. The evaluation in the post-natal period was made with US scans at 15, 45 days, and 5 months after birth.

31 patients underwent surgery: 6 with simple cysts, 24 complex cysts and 1 symptomatic.

Sixteen of these cysts were detected in the right ovary and 15 in the left ovary. In 3 cases there was a free floating cyst (detected as left auto-amputated ovary).

Six cases of simple cysts required surgery. Five patients underwent surgery due to persistence of the cyst at further US examinations and 1 patient due to the onset of important abdominal pain ( 2 years old baby lost to follow-up). In this case we found evidences of ovarian and tubal torsion.

All 24 complex cysts diagnosed with the first post-natal US scan underwent surgery. Intraoperative context showed evidences of ovarian and tubal torsion in $23 / 24$ cases.

Mean diameter of simple and complex cysts before surgery was respectively 6,5 $(5 \cdot 0-9.0 \mathrm{~cm})$ and $5,5 \mathrm{~cm}(4.3-7.0 \mathrm{~cm})(\mathrm{p}>0,05)$. Mean age at surgery was respectively 38,8 and 38,6 days $(p>0,05)$ for simple and complex cysts.

In 19 cases the authors performed standard laparoscopy, and in 12 cases trans-umbilical laparoscopic-assisted procedure; in 29 cases, authors performed puncture and percutaneous evacuation of the cyst under vision, using a fine needle.

Twenty patients were treated with sparing surgery: 11 cystectomy and 9 marsupialization. No cases had oophoropexy, nor of the controlateral ovary. Total oophorectomy was realized in 11 cases.

\section{All patients had a normal post-operative course.}

During follow-up, about the conservative treatment surgical subgroup, 11 cases $(73,3 \%)$ had detectable residual ovary; in 4 cases there was no evidence of residual ovary; 5 patients were lost at followup.

\section{Discussion}

Prenatal US scans show many foetal ovarian masses that in the previous years would have gone unrecognized [6].

Early ovarian cysts are usually follicular cysts: the foetal ovary is stimulated by foetal gonadotropins, maternal estrogens and placental chorionic gonadotropin; at birth levels of gonadotropins rise rapidly due to the decrease in oestrogen and progesteron levels. In diabetic, pre-eclamptic or isoimmunized mothers' newborns occur more frequently luteinic cysts [7-8].

The effect of gonadotropins is significant in the first few months of life, thereafter the gonadotropins levels decrease and most of the cysts resolve; spontaneous resolution of simple ovarian cysts is expected within 6 months of age [9].

US scan represent the gold standard for diagnosis. The diagnosis or the suspicion of foetal ovarian cysts is based on two unspecific ultrasound criteria: presence of a cystic structure of regular size in the lower and lateral side of the abdomen and regular appearance of the urinary and gastrointestinal tracts [10].

Nussbaum's classification has reached a general consensus and any change of the sonographic pattern from purely cystic to an echogenic or mixed pattern is considered as the onset of a complication, for example torsion, rupture, auto-amputation or haemorrhage.

Torsion is the most common complication reported with an incidence of $30-78 \%$ of all neonatal ovarian cysts [11-12].

In addition to the typical ultrasound findings, it's important to consider the "daughter cyst sign", which is defined as a visualization of small cysts along the cyst wall (representing follicles), as a specific sonographic finding for an uncomplicated ovarian cyst [12].

It is our experience and opinion that there is no necessity for other diagnostic techniques while studying these patients; even if it is suggested by others to perform MRI or videolaparoscopy to differentiate ovarian cysts from other abdominal masses, but both requires general anesthesia, while only videolaparoscopy is both a diagnostic and therapeutic procedure.

Otherwise the authors feel confident in suggesting the use of US scan as a suitable method to monitor cystic formation; it is described in literature that MRI provides better results for ovarian cysts with a diameter $<6 \mathrm{~cm}[10]$. 
Citation: Camoglio FS, Bianchi F, Peretti M, Patanè S, Spigo V, et al. (2017) Management of Neonatal Ovarian Cysts: Clinical Aspects. Int J Gynecol Clin Pract 4: 130. doi: https://doi.org/10.15344/2394-4986/2017/130

Page 3 of 3

In general, postnatal choice of treatment depends on the size and appearance of the cyst. A wait and see policy is preferred due to the high percentage of spontaneous resolution for simple cyst $<5 \mathrm{~cm}$ [13].

Surgical treatment is indicated only in complex cysts or in simple cysts $>5 \mathrm{~cm}$ in order to avoid further complications and to preserve residual ovary, especially in presence of bilateral cysts [14-15].

Complex cysts usually do not require urgent surgery because they generally represent the outcome of an antenatal ovarian torsion.

If compared with traditional surgery, minimally invasive technique is the gold standard. Laparoscopy reduces invasive surgery to a minimum, allowing an ideal post-operative course: fast physiological recovery, minimum pain and short hospitalization.

An innovative approach to surgical treatment of ovarian cyst is the laparoscopic-assisted transumbilical ovarian cystectomy; it is a relatively simple procedure, which combines laparoscopy and traditional extracorporeal surgery and that appears to have the advantages of laparoscopy, such as excellent visualization of the controlateral ovary, and excellent cosmesis, with only a single incision. In addition to that, these techniques can be performable as a day surgery procedure for patients older than 6 months [16-17].

\section{Conclusion}

Ovarian cysts in neonates are uncommon but their number is increasing in the latest years; these patients need US follow-up based on cysts' diameters. In these patient MRI is not indicated (especially in large ovarian cyst) When indicated minimally invasive surgery is the first choice. These procedures in selected patients could be performed with short hospitalization such as day surgery admission.

\section{Competing Interests}

The authors declare that they have no competing interests.

\section{References}

1. Chandler JC, Gauderer MW (2004) The neonate with an abdominal mass. Pediatr Clin North Am 51: 979-997.

2. Bryant AE, Laufer MR (2004) Fetal ovarian cysts: incidence, diagnosis and management. J Reprod Med 49: 329-337.

3. Nussbaum AR, Sanders RC, Hartman DS, Dudgeon DL, Parmley TH (1988) Neonatal ovarian cysts: sonographic-pathologic correlation. Radiology 68 : 817-821.

4. Zampieri N, Scirè G, Zamboni C, Ottolenghi A, Camoglio FS (2009) Unusual presentation of antenatal ovarian torsion: free-floating abdominal cysts. Our experience and surgical management. J Laparoendosc Adv Surg Tech A 1: S149-S152.

5. Bagolan P, Rivosecchi M, Giorlandino C, Bilancioni E, Nahom A, et al. (1992) Prenatal diagnosis and clinical outcome of ovarian cysts. J Pediatr Surg 27: 879-881.

6. Dolgin SE (2000) Ovarian masses in the newborn. Semin Pediatr Surg 37: 25-28.

7. Defoort $\mathrm{P}$, Thiery M, Derom R, Vanhaesebrouk $\mathrm{P}$, de Praetere $\mathrm{C}$, et al (1990) Ovarian cysts in the fetus and neonate. $Z$ Geburtsh u Perinat 194: 137-139.

8. Ahlvis RC, Bauer WC (1957) Luteinised cysts in the ovaries of infants born of diabetic mothers. Am J Dis Child 93:107-111.

9. Nussbaum AR, Sanders RC, Benator RM, Haller JA, Dudgeon DL (1987) Spontaneous resolution of neonatal ovarian cysts. Am J Res 148:176-178.
10. Zampieri N, Borruto F, Zamboni C, Camoglio FS (2008) Foetal and neonatal ovarian cysts: a 5-year experience. Arch Gynecol Obstet 277: 303-306.

11. Cass DI (2005) Ovarian torsion. Semin Pediatr Surg 14: 86-91.

12. Lee HJ, Woo SK, Kim JS, Suh SJ (2000) "Daughter cyst" sign: a sonographic finding of ovarian cyst neonates, infants and young children. AJR Am J Roentgenol 174: 1013-1015.

13. Ozcan HN, Balci S, Ekinci S, Gunes A, Oguz B, et al. (2015) Imaging Findings of Fetal-Neonatal Ovarian Cysts Complicated With Ovarian Torsion and Autoamputation. AJR Am J Roentgenol 205: 185-189.

14. Sapin E, Bargy F, Lewin F, Baron JM, Adamsbaum C, Barbet JP, et al (1994) Management of ovarian cyst detected by prenatal ultrasounds. Eur J Pediatr Surg 4: 137-140.

15. Heling KS, Chaoi R, Kirchmair F, Stadie S, Bollmann R (2002) Fetal ovarian cysts: prenatal diagnosis, management and postnatal outcome. Ultrasound Obstet Gynecol 20: 47-52.

16. Prasad S, Chui $\mathrm{CH}$ (2007) Laparoscopic-assisted transumbilical ovarian cystectomy in a neonate. JSLS 11: 138-141.

17. Schenkman L, Weiner TM, Phillips JD (2008) Evolution of the surgical management of neonatal ovarian cysts: laparoscopic-assistedtransumbilical extracorporeal ovarian cystectomy (LATEC). J Laparoendosc Adv Surg Tech A 18: 635-640. 